\title{
Preparation of a Doxorubicin Conjugate with (Co) Polymers of Methacrylic acid for Targeted Tumor Therapy
}

\author{
Olga Zhukova ${ }^{1 *}$, Svetlana Bulgakova ${ }^{2}$ \\ ${ }^{1}$ Nizhny Novgorod State Medical Academy, Nizhny Novgorod, Russia. \\ ${ }^{2}$ Lobachevsky State University of Nizhny Novgorod, Nizhny Novgorod, Russia.
}

\begin{tabular}{|c|c|}
\hline ARTICLE INFO & ABSTRACT \\
\hline $\begin{array}{l}\text { Article history: } \\
\text { Received on: 08/02/2016 } \\
\text { Revised on: } 11 / 04 / 2016 \\
\text { Accepted on: } 06 / 05 / 2016 \\
\text { Available online: } 28 / 07 / 2016\end{array}$ & $\begin{array}{l}\text { This study was performed in order to creation of a doxorubicin conjugate with methacrylic acid (co)polymers } \\
\text { for targeted tumor therapy. Poly }(t \text {-butylmethacrylate) with the optimal for biocompatible polymers molecular- } \\
\text { weight characteristics }\left(M_{\mathrm{n}}=12,400 ; M_{w} / M_{\mathrm{n}}=1.35\right) \text { was synthesized by radical polymerization in the presence of } \\
\text { thioglycolic acid as a chain transfer agent. The obtained polymer was converted by acid hydrolysis into a water- } \\
\text { soluble copolymer of } t \text {-butylmethacrylate and methacrylic acid of } 20: 80 \text { mass } \% \text {, respectively. The copolymer of }\end{array}$ \\
\hline $\begin{array}{l}\text { Key words: } \\
\text { polymeric drug carrier, } \\
\text { copolymer of methacrylic } \\
\text { acid and } t \text {-butylmethacrylate, } \\
\text { doxorubicin, folate vector, } \\
\text { UV-spectroscopy, IR- } \\
\text { spectroscopy, NMR- } \\
\text { spectroscopy. }\end{array}$ & $\begin{array}{l}\text { conjugate of the copolymer with doxorubicin and also a conjugate with the folate vector were synthesized. Their } \\
\text { formation was proved using elemental analysis and IR, UV, NMR spectroscopy. Thus, in the course of the study } \\
\text { the conjugate of doxorubicin was synthesized, combining the drug and a vector for delivery of the drug. This } \\
\text { compound is a promising, as it represents a new form of transport delivering a well-known and widely used } \\
\text { anticancer agent doxorubicin. }\end{array}$ \\
\hline
\end{tabular}

\section{INTRODUCTION}

Currently, the pharmacotherapy of cancer has a large volume of high-performance drugs, but treatment treatable results often remain unsatisfactory. Among the factors that reduce the effectiveness of drugs, a significant place is occupied by unfavorable biodistribution, which may be due to difficulties in penetrating into the pathological process place. A common way to overcome this obstacle and improve the therapy effectiveness is to increase the dose of drugs. However, the therapeutic effect is often achieved at the cost of increasing nonspecific toxicity. This problem is particularly acute in the case of anticancer drugs whose use is associated with side effects

* Corresponding Author

Email:ov-zhukova@mail.ru significantly reducing the therapeutic value of drugs themselves. In recent decades the new methods of anticancer therapy are actively developed. One of the most promising developments in this area is the transport systems of selective drug delivery. The polymer compounds are widely used as the carriers because of their ability to modify the pharmacokinetics of drugs due to the high of the molecular weight (MW) that results in increasing the circulating of the drugs in a blood and the capture process (endocytosis) polymer derived by the tumor cells. In addition, many primary and metastatic tumors have a discontinuous and high-permeability of vessels system that also defines an increased uptake of drugs on a polymer carrier by tumor cells (Gabizon, 2006).

One way of creating such drugs is the design of complex polymer systems containing "recognizing" components (vectors). The folic acid is a water-soluble vitamin and is a ligand to folate receptors, increased expression of which is characteristic of tumor cells, can be used as one of such vectors (Xu et al., 2013). 
Folate receptor is an attached glycoprotein to a glycosylphosphatidylinositol that binds with folic acid and it's internalizing into the cells by receptor-mediated endocytosis (Salazar and Ratnam, 2007). Obviously, the use of folic acid as a vector for tumor tissue is a promising direction in antitumor therapy.

Polymer systems of selective delivery of anticancer drugs are widely studied in different directions (Nori et al., 2003; Gabizon et al., 2003; Yoo et al., 2000). There are polymer nanogels able to reversibly responding to small external effects (temperature, ionic strength, $\mathrm{pH}$, electric field, etc.), as well as to include in its volume and controllably release the active drug substance, and the amphiphilic polymer micelles the core of which is the drug (Bae et al., 2007; Adams et al., 2003; Gaucher et al., 2010; Duncan, 2006). Another method includes the chemically binding the drug to a carrier to form a physiologically active polymer, releasing the drug on hydrolysis in cells ( $\mathrm{Li}$ and Wallace, 2008). It should be noted that the majority of researchers in the search of polymers with the purpose of immobilization-based drugs, stop the focus on the biopolymers. In this regard, there is a tendency to replace biopolymers on available synthetic analogues, whose properties are often not inferior, and sometimes superior to the biopolymers. The methacrylic copolymers are among of the synthetic polymers. Thus, they were used to create a series of delivery transport systems of anticancer drugs (Wang et al., 2010; Bayazit et al., 2010; Gann et al., 2009). For example, it is known, that the $\mathrm{pH}$-sensitive hydrogels based on polyacrylic acid have good biocompatibility. Furthermore, it is known, that poly(met)acrylic acids themselves possess antitumor and antiviral properties, as determined by the values of their molecular weight and the molecular weight distribution. It turned out that when using the (co)polymers their overall toxicity to the organism is significantly reduced, that it is especially important in the context of anticancer therapy.

Thus, commercial products are frequently used, which limits the choice of molecular weight characteristics of the polymer matrix. As for (meth)acrylic polymers, then today very narrow circle of their derivatives are offered to study as carriers of anticancer agents.

\section{MATERIALS AND METHODS}

\section{Materials}

For synthesis of the polymer carrier the following reagents were used: tert-butyl methacrylate (TBMA) as a monomer (98\%, Aldrich); folic acid (FA) (97\%, Fluka), Nhydroxysuccinimide (NHS) (98\%, Fluka), dicyclohexylcarbodiimide (DCC) (98\%, Alfa Aeser), doxorubicin (DOX), thioglycolic acid (TGA), hexamethylenediamine (HMDA), hydrazine hydrate and azobisisobutyronitrile (AIBN) as a radical polymerization initiator. AIBN was purified by recrystallization from isopropyl alcohol and dried in a vacuum oven to constant weight. Reagents purchased from catalogs were not subjected to additional purification. HMDA was purified by sublimation and TGA - by distillation in vacuo. Solvents such as methanol, dioxane, tetrahydrofuran (THF), dimethylsulfoxide (DMSO) were purified by standard techniques (Weissberger et al., 1955).

\section{Preparation of conjugate of poly(tert-butyl methacrylate) (PTBMA)}

TBMA polymerization was carried out in bulk at $70^{\circ} \mathrm{C}$ in the presence of $5 \cdot 10^{-2} \mathrm{M}$ AIBN. TGA was used as the chain transfer agent. Initial reaction mixture was charged into an ampoule and refreezing previously degassed under vacuum three times, than the vials were sealed. The polymer was purified by reprecipitation three times from acetone into water, and then dried in vacuo to constant weight.

Synthesis of folate vector (FV) was conducted by carbodiimide method. To a solution of $0.44 \mathrm{~g}$ of FA $(1 \mathrm{mmol})$ in DMSO $(30 \mathrm{ml})$ was added $0.206 \mathrm{~g}$ DCC $(1 \mathrm{mmol})$ and $0.17 \mathrm{~g}$ NHS ( $1.5 \mathrm{mmol})$. The reaction was conducted in the dark with stirring for 6 hours at room temperature. After the reaction, the precipitate of dicyclohexylurea was separated, and to the reaction mixture was added $0.12 \mathrm{~g}$ of HMDA ( $1 \mathrm{mmol})$. The mixture was left overnight at room temperature, and then the excess acetonitrile was added. The precipitate was centrifuged, washed three times with diethyl ether $(10 \mathrm{ml})$ and then dried in a vacuum oven to constant weight. The yield was $84 \%$. To attach FV to PTBMA, at the beginning PTBMA was modified by carbodiimide method with the formation of polymer having NHS - end groups.

Hydrolysis of PTBMA was carried out in a medium dioxane at the presence dilute $(1: 2) \mathrm{HCl}$ in the amount of $0.5 \mathrm{ml}$ per $0.25 \mathrm{~g}$ of the polymer at a temperature of $60^{\circ} \mathrm{C}, 80^{\circ} \mathrm{C}, 100^{\circ} \mathrm{C}$ to reflux. The content of the methacrylic acid (MAA) was determined by potentiometric acid-base titration in a mixture of methanol and THF.

The synthesis of the polymer derivative DOX was performed by the carbodiimide method. First the activation of copolymer TBMA-MAA=20:80 by wt. \% prepared by hydrolysis of PTBMA was performed. To a solution of $0.075 \mathrm{~g}$ of the copolymer in DMSO $0.0043 \mathrm{~g}$ of DCC $(0.02 \mathrm{mmol})$ and $0.0036 \mathrm{~g}$ of NHS $(0.03 \mathrm{mmol})$ was added. The mixture was placed in a refrigerator for 12 hours. Then the precipitate of dicyclohexylurea was separated and to the solution $0.01 \mathrm{ml}$ of hydrazine hydrate (0.2 mmol) was added. The reaction was conducted in the dark with stirring for 6 hours at room temperature. To the reaction mixture $0.01 \mathrm{~g}$ of DOX $(0.02 \mathrm{mmol})$ previously dissolved in methanol and acetic acid as an acidic activator were added. The mixture was left for 48 hours in a dark place. Modified polymer was precipitated by acetonitrile -acetone (3:1) mixture, and the precipitate was centrifuged, washed three times with acetonitrile and dried in a vacuum oven.

\section{Determination of the molecular weight characteristics of the polymer}

Molecular weight characteristics of the polymers were determined via gel permeation chromatography (GPC) in THF at 
$40^{\circ} \mathrm{C}$ relative to polymethylmethacrylate standards. Measurements were performed on a Prominence LC-20VP (Shimadzu) liquid chromatograph equipped with two columns packed with styrogel (pore sizes of $10^{6}$ and $10^{5} \AA$ ) and a differential refractometer.

\section{IR - Spectroscopy}

The registration of the IR-spectra of the compounds were carried out on a IR-Fourier spectrometer FSM 1201 (Ltd. "Monitoring", St. Petersburg) in the spectral range from 400 to $4000 \mathrm{~cm}^{-1}$ (resolution of $4 \mathrm{~cm}^{-1}$, the number of scans $32 ; \mathrm{KBr}$ disk). Samples were prepared as a suspension in vaseline oil.

\section{UV - Spectroscopy}

UV-spectra of the samples were recorded on a UVspectrophotometer Shimadzu UV 1650 DC (190-1100 nm spectral range, wavelength error of $0.3 \mathrm{~nm}$ ). The solutions of compounds were prepared in DMSO.

\section{NMR Spectroscopy}

${ }^{1} \mathrm{H}$ nuclear magnetic resonance (NMR) spectra were recorded on a Bruker FT-80 spectrometer (Bruker) instrument operating at a frequency of $400 \mathrm{MHz}$ and chemical shifts $(\delta)$ are in parts per million (ppm) relative to internal tetramethylsilane. To this end, $\mathrm{CDCl}_{3}$ was used as the solvent for PTBMA, while doxorubicin, copolymer TBMA-MAA, conjugate of copolymer with doxorubicin were dissolved in $\left(\mathrm{d}_{6}\right)$ DMSO.

\section{Elemental Analysis}

The elemental analysis was carried out using elemental analyzer vario $\mathrm{EL}$ cube for the simultaneous determination of $\mathrm{C}$, $\mathrm{H}, \mathrm{N}, \mathrm{S}$.

\section{Determining the degree of binding of doxorubicin to the copolymer}

The degree of doxorubicin attachment to the copolymer TBMA-MAA-FV was determined by photometrically on a spectrophotometer UV mini-1240 Shimadzu. For this purpose it was obtained a calibration graph of optical density of solutions of doxorubicin on concentration at $\lambda=490 \mathrm{~nm}$. Then on the optical density of the investigated solution (supernatant) and a calibration curve the desired concentration of unreacted DOX was found.

Then, the degree of doxorubicin attachment to the polymer was determined with according to the formula (1).

$$
P=\frac{m_{0}-m_{x}}{m_{0}} \times 100
$$

where $P$ - the degree of grafting DOX (\%), $m_{0}-$ the mass of the entire DOX $(\mathrm{mg}), m_{\mathrm{x}}$ - the mass of unbound DOX $(\mathrm{mg})$.

\section{Determining the degree of DOX release from polymer carrier in vitro}

Determining the degree of DOX release was performed in a buffer solution at $\mathrm{pH} 4.8$ and 7.4 in the dialyzer, whose cells are separated by a membrane CelluSep T1, with MW of 3500 daltons.

The polymer conjugate was dissolved in a buffer solution $(2 \mathrm{mg} / \mathrm{ml})$ and placed in one of the dialyzer cells. The appropriate buffer solution was placed into other cell. Thereafter, the dialyzer was placed in a thermostat at $37^{\circ} \mathrm{C}$. Through certain time intervals all the contents of the cell with the buffer solution was sampled for determination of free DOX and then this cell was filled with fresh solution.

\section{RESULTS AND DISCUSSION}

Selection of PTBMA as the polymeric carrier is connected with the possibility of hydrolysis to form polymethacrylic acid, which is a biocompatible polymer. Since the biopolymers have to meet certain requirements in MW not exceeding the renal threshold $M_{\mathrm{n}} \leq 30,000$, for the regulation of this parameter it was used a chain transfer reaction to TGA, as a result of which the macromolecules of PTBMA assume the end carboxyl functionality required for the modification of the polymer with folate vector (Figure 1).

It is important to know the kinetic parameters of polymerization of TBMA in the presence of TGA for the directed synthesis of the polymer with the desired characteristics. To this end, the influence of TGA concentration as on the initial rate and on the polymerization degree was studied in the presence of $0.05 \mathrm{M}$ AIBN as a radical initiator.

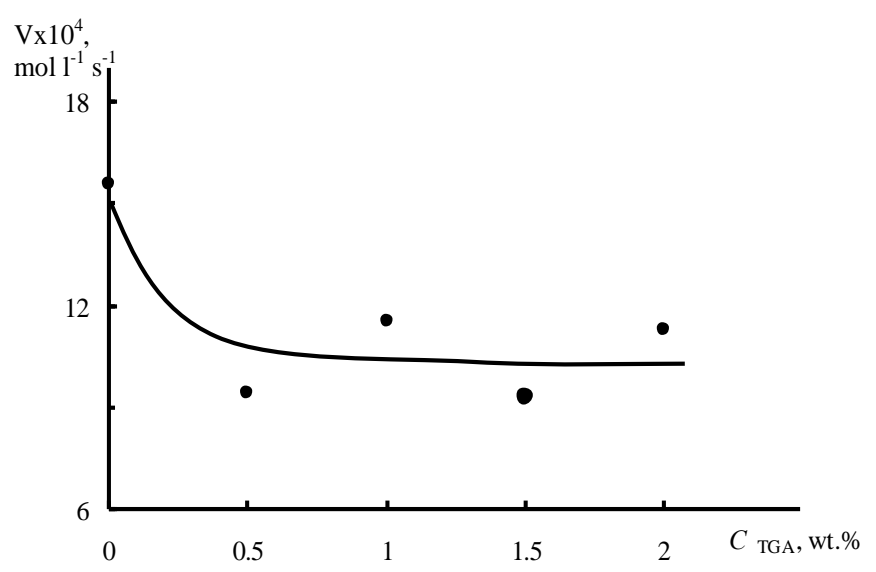

Fig. 1: The dependence of the initial rate of TBMA polymerization in bulk on the TGA concentration, $($ AIBN $)=0.05 \mathrm{M}, 70^{\circ} \mathrm{C}$

As can be seen from Fig. 1, the polymerization rate of TBMA decreases in 1.5 times already with the introduction of $0.5 \%$ TGA and then is almost independent on the concentration of chain transfer agent.

The decrease in polymerization rate is due to the fact that the radical $\mathrm{HOOCCH}_{2} \mathrm{~S}$. formed by chain transfer reaction, is more stable owing to the lone-electron pair of the sulfur atom, and therefore less active than the TBMA radical. 

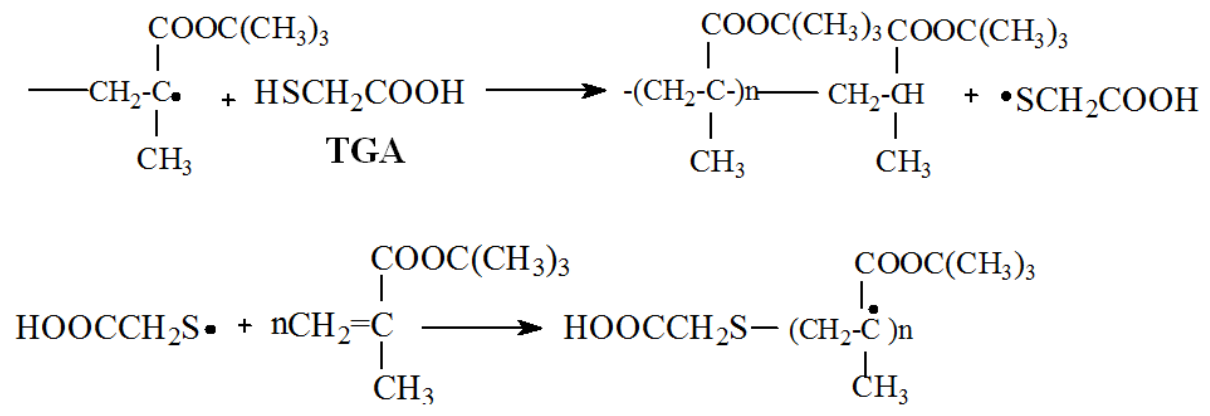

TBMA

Scheme 1. The chain transfer reaction on TGA at TBMA radical polymerization.

Changing the degree of polymerization of the polymer by increasing the concentration of THC in the absence of chain transfer reactions to the polymer, the monomer and the initiator is described by equation Mayo:

$$
\frac{1}{\overline{P_{n}}}=\frac{1}{P_{0}}+C_{S} \frac{[S]}{[M]}
$$

where $C_{\text {s }}$ - chain transfer constant, $\bar{P}_{n}$ И $\overline{P_{0}}-$ the average degree of polymerization in the absence and the presence of TGA, respectively; $(S),(M)$ - the mole concentration of TGA and TBMA, respectively.

From the slope of the line on the graph depending shown in Fig. 2, the constant of chain transfer to TGA $C_{\mathrm{s}}=1.92$ was calculated.

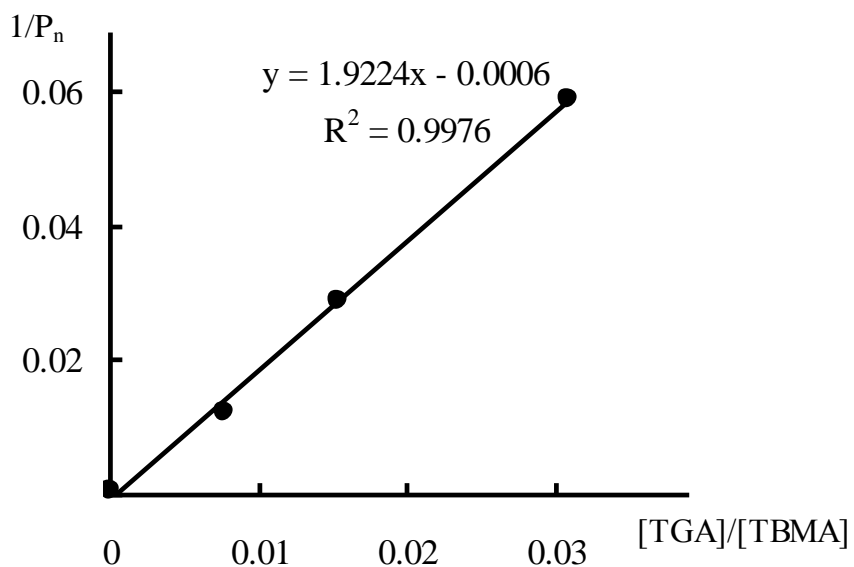

Fig. 2: The Mayo`s dependence at bulk polymerization of TBMA in the presence of TGA, $(\mathrm{AIBN})=0.05 \mathrm{M}, 70^{\circ} \mathrm{C}$

Knowing this value it is possible to predict with a high probability the MW polymer depending on the chain transfer agent concentration during the polymerization to deep conversion and evaluate the effectiveness of the end polymer group's functionalization.

Using the obtained results, the concentration of TGA was selected equal to $1 \mathrm{wt} . \%$, at which conversion is attained 98\% for 4 hours, and PTBMA is formed with optimal for biocompatible polymer characteristics such as $M_{\mathrm{n}}=12400$ and the polydispersity index $M_{\mathrm{w}} / M_{\mathrm{n}}=1.35$.

Simple theoretical calculation was applied for estimating the degree of functionalization of the polymer by terminal carboxyl groups under the assumption that the constant of chain transfer will not change during the entire polymerization process. Its essence consists in the following. Based on the proportions that one mole TGA spends on $x$ mole TBMA, and $\left((S)_{0}-(S)\right)$ mole TGA - on $\left((M)_{0}-(M)\right)$ mole TBMA, we obtain: $x=(M)_{0}-(M) /\left((S)_{0}-(S)\right)$, in particular for the conversion of $98 \%, x=0.98(M)_{0} /\left((S)_{0}-(S)\right)$. The unknown concentration of chain transfer agent $(S)$ in this equation can be calculated from equation (2) for this conversion:

$$
\frac{[S]}{[S]_{0}}=\left(\frac{[M]}{[M]_{0}}\right)^{C_{S}}
$$

At given values of $(M)_{0}$ and $(S)_{0}$, (bulk polymerization of TBMA, $1 \%$ TGA) by equation (2), we find that $x=70$ mole TBMA. It means that for every 70 units of TBMA there is one residue of TGA. In our case at $M_{\mathrm{n}}=12400$ each macromolecule contains 12400:142 $=87$ TBMA repeating units, i.e. we can assume that almost all macromolecules PTBMA are functionalized by the carboxyl groups. It is also confirmed by elemental analysis (Table 1).

Table 1. Results of elemental analysis of the sample PTBMA

\begin{tabular}{lllllll}
\hline \multicolumn{7}{c}{ The content of the element, \%* } \\
\hline $\mathrm{N}$ & $\mathrm{C}_{\exp }$ & $\mathrm{C}_{\text {theor }}$ & $\mathrm{H}_{\exp }$ & $\mathrm{H}_{\text {theor }}$ & $\mathrm{S}_{\exp }$ & $\mathrm{S}_{\text {theor }}$ \\
\hline 0 & $65.99 \pm 0.04$ & 67.6 & $10.19 \pm 0.33$ & 9.86 & $0.28 \pm 0.05$ & 0.26 \\
\hline
\end{tabular}

*The theoretical content of elements in the PTBA sample was calculated by the formula:

$$
\begin{aligned}
X_{\text {theor }}= & \frac{M_{\text {element }}}{142} \times 100 \% \\
\mathrm{~S}_{\text {theor }}= & \frac{32}{12400} \times 100 \%
\end{aligned} \text {, and sulfur by the formula: }
$$

The presence of the free carboxyl group was used for modification of the polymer with folic acid, which is used as a vector to tumor cells. Folate vector directly was prepared by carbodiimide method according to the scheme described in 
(Zhukova and Bulgakova, 2015). In the next stage the hydrolysis of the obtained PCBMA-PV was carried out in an acidic medium. The degree of hydrolysis of the PTBMA was reached $80 \%$ for 12 hours at $100^{\circ} \mathrm{C}$, i.e. the copolymer of TBMA-MAA $=20: 80 \mathrm{wt} . \%$, containing the PV end-functionality was produced.

The data of NMR-spectroscopy confirm of these chemical reactions (Fig. 3). In the NMR-spectrum of the copolymer modified by FV the peaks appear at 1.23, $1.26 \mathrm{ppm}$, which are observed in the NMR-spectrum of FV and missing in the spectrum of the "pure" copolymer. In the spectrum of FV the peaks are recorded at $2.36,2.39$ and $2.40 \mathrm{ppm}$, and at 7.12, 7.22, $7.34 \mathrm{ppm}$, which take place in the spectrum of the modified copolymer $(2.38,2.39,2.41$ and $7.02 \mathrm{ppm}, 7.15,7.28 \mathrm{ppm}$, respectively). 1a

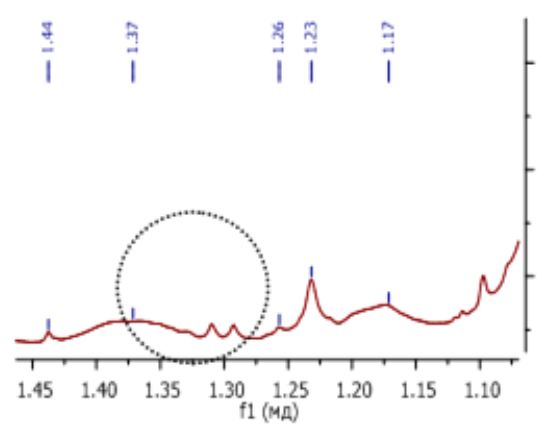

1b

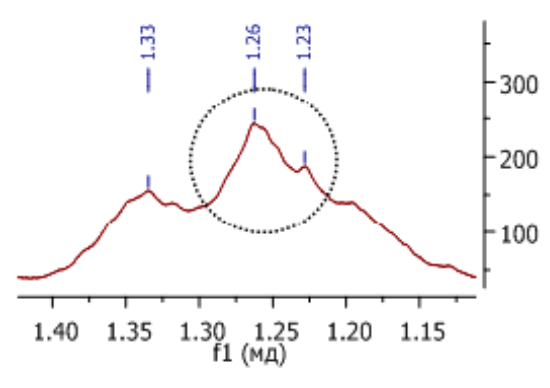

2a

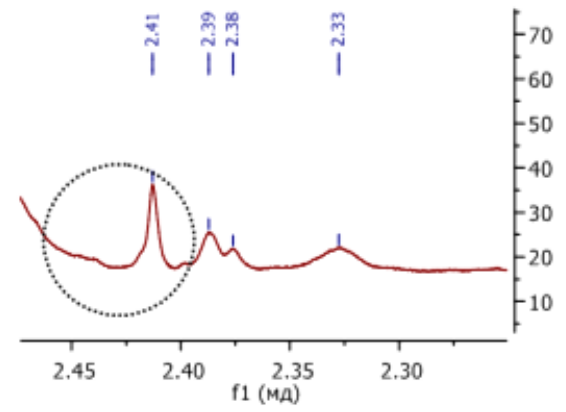

2b
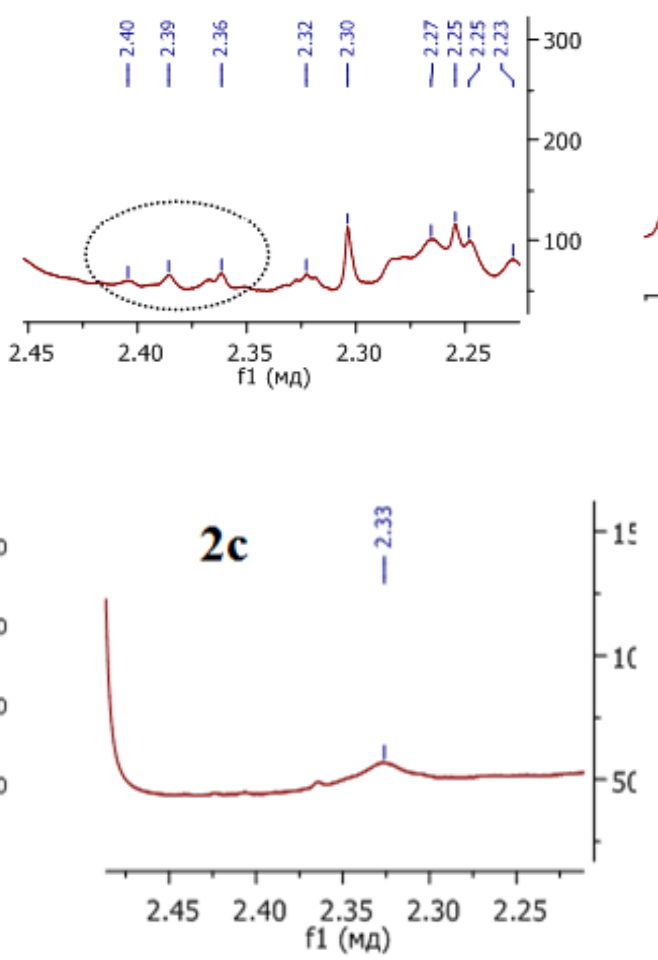

$3 a$

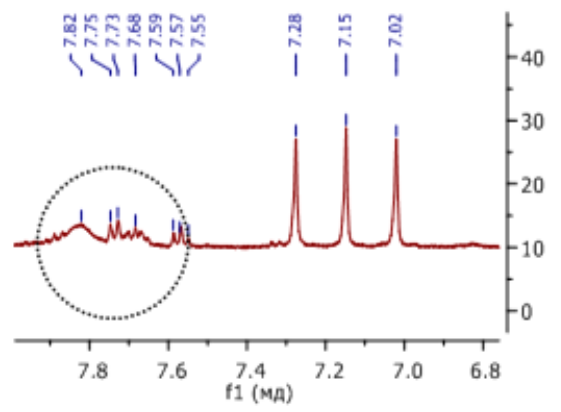

$3 \mathrm{~b}$

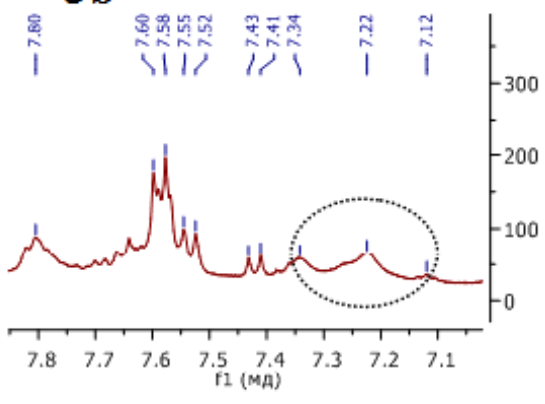

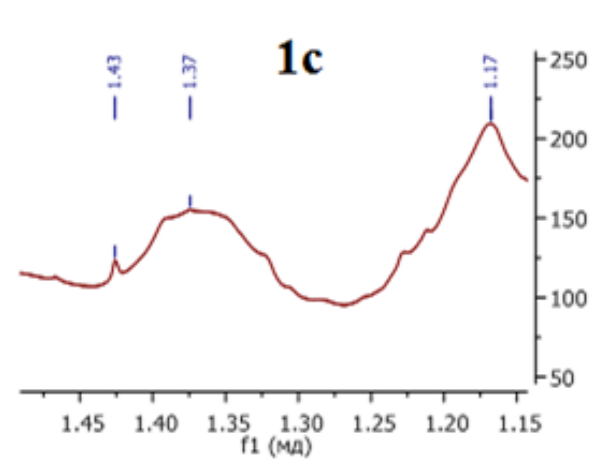

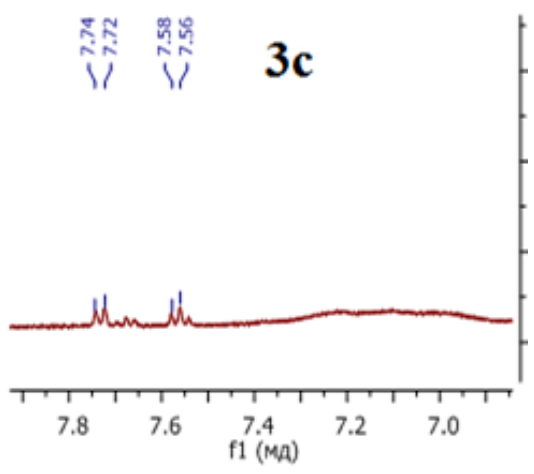

Fig. 3: NMR- spectra fragments of TBMA-MAA-FV (a), TBMA-MAA (b), FV (c) in the field of $\delta=1.2-1.3$ ppm (1); $2.25-2.45$ ppm (2); 7 - 8 ppm (3) 
A number of polymer-analogic transformations of DOX were performed before it was chemically grafted to TBMA-MAA copolymer (20:80 wt.\%). First, a part of the MAA carboxylic units in the copolymer ( $3-5$ mol. \%) were converted to NHS groups. Then, these groups were modified by hydrazine to form hydrazides, after which DOX was conjugated as shown in Scheme 2 .

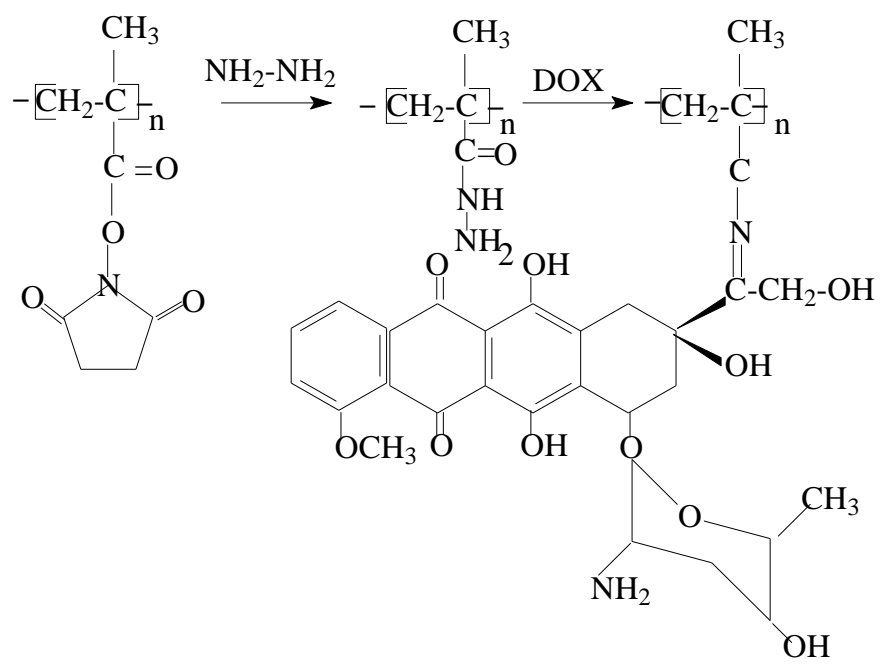

Scheme 2. Chemical grafting of DOX to hydrazine-modified MAA unites.

The data UV-, IR-, NMR-spectroscopy are an evidence of the formation of a conjugate having in its composition of $\mathrm{FV}$ and DOX. As can be seen from Figure 4, in the UV-spectrum of the polymer conjugate (TBMA-MAA)-DOX-FV pronounced peak at $\lambda=289 \mathrm{~nm}$ appears, which is typical for FV and FA. This peak absent in the spectra of the compounds not having of FA, namely, DOX (curve 1) and a copolymer of TBMA-MAA = 20: $80 \mathrm{wt} . \%$ (Curve 2).

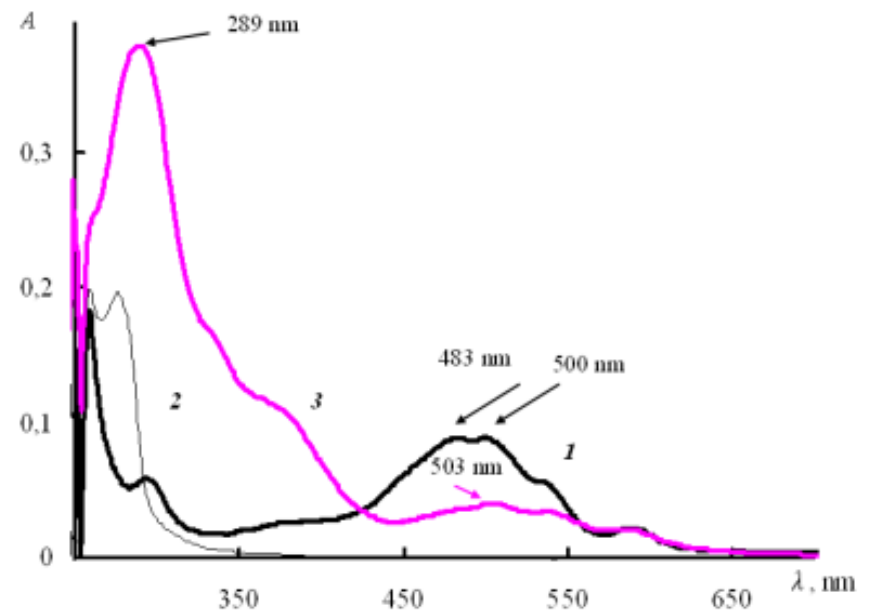

Fig. 4. UV-spectra of DOX (1), TBMA-MAA $=20: 80$ wt.\%) copolymer (2), and (TBMA-MAA)-DOX-FV conjugate (3) in DMSO solutions $\left(C=4 \cdot 10^{-7}\right.$ $\mathrm{g} / \mathrm{ml})$

DOX is known to have a broad characteristic band in the range of $\lambda=480-500 \mathrm{~nm}$. The maximum can be observed at $480 \mathrm{~nm}$ and
$490 \mathrm{~nm}$ depending on the solvent and $\mathrm{pH}$. The DOX sample in DMSO that was analyzed has a broad band in the range $\lambda=480$ $500 \mathrm{~nm}$ with maxima at 483 and $500 \mathrm{~nm}$. A broad peak with a maximum at $\lambda=503 \mathrm{~nm}$ (bathochromic shift) is also in the UVspectrum of the polymeric derivative of DOX.

The shifts of these maxima were explained by the formation of a hydrazone bond between the polymeric hydrazide derivative and the tetracyclic anthraquinoid aglycon of doxorubinon. In the IR-spectrum of the DOX polymer conjugate (Figure 5, curve 1) the peaks are registered of $1616 \mathrm{sm}^{-1}$ and 1580 $\mathrm{cm}^{-1}$, which are shown in DOX and missing in the spectra of copolymer. The peak at $1616 \mathrm{sm}^{-1}$ is also typical for $\mathrm{C}=\mathrm{N}$ hydrazone bond. In the spectrum of the conjugate amide band at $1547 \mathrm{sm}^{-1}$ appears which is not present in the IR-spectrum of the copolymer and DOX. It is also confirmed of chemical bonding between the polymer and DOX.

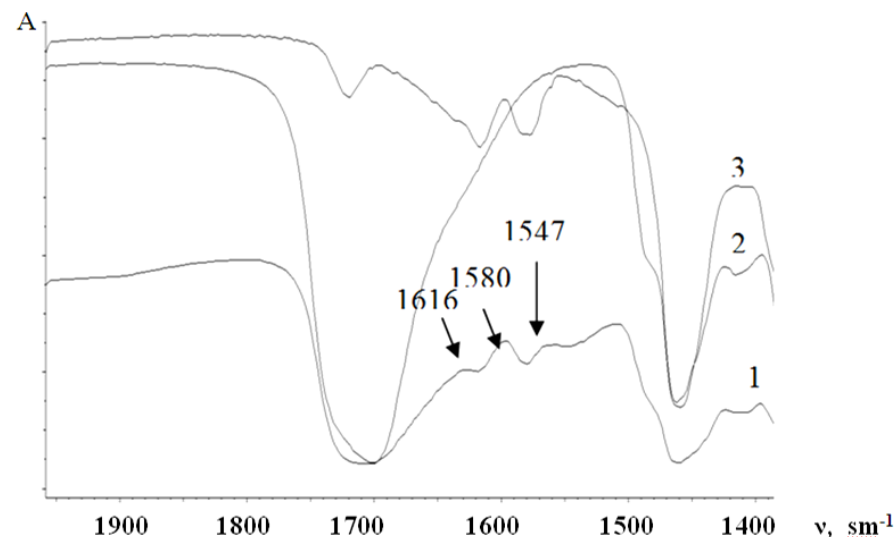

Fig. 5. IR-spectra of polymeric DOX derivative (1), TBMA-MAA copolymer (2), and DOX (3).

As confirmation of formation of the conjugate copolymer TBMA-MAA-FV with DOX are data of NMR-spectra (Figure 6). Curve of NMR-spectrum of the conjugate in $3.35-3.62 \mathrm{ppm}$ repeats the NMR-spectrum of DOX. The degree of binding to the polymer DOX was $62.7 \pm 11.8 \%$.

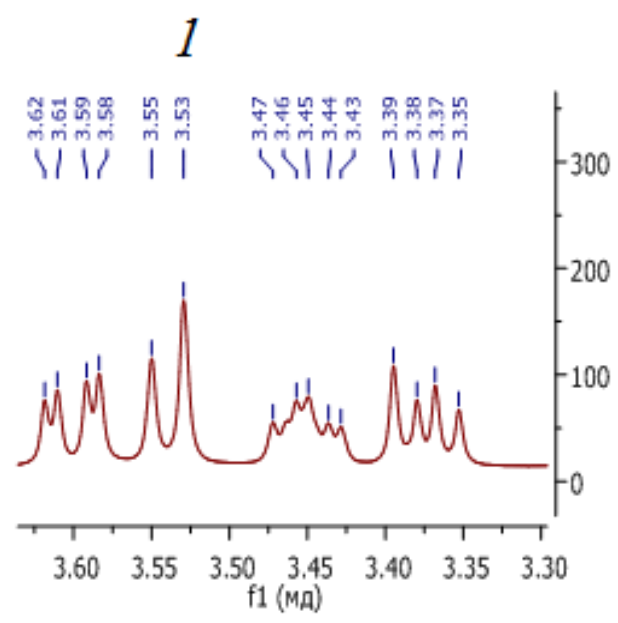

Fig. 6: continued... 

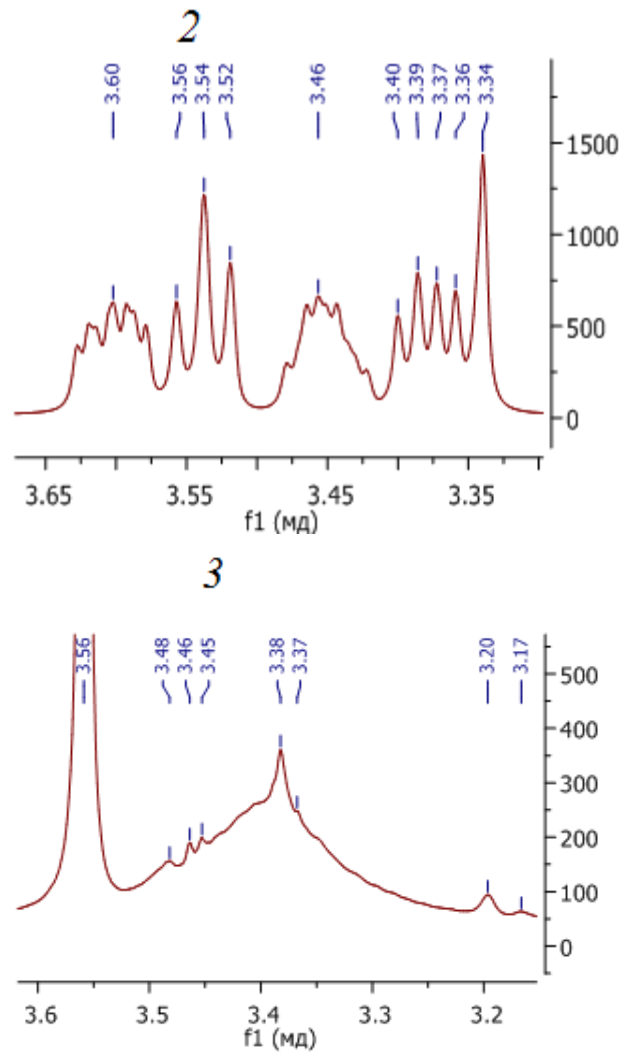

Fig. 6: NMR-spectra fragments of TBMA-MAA-FV-DOX (1), DOX (2), TBMA-MAA (3)

Conducted research of determining of the DOX release extent from polymeric derivate in medium under conditions, imitating of biological media, showed that the drug release is faster almost 3 times at $\mathrm{pH} 4.6$ than at $\mathrm{pH} 7.4$ (Figure 7).

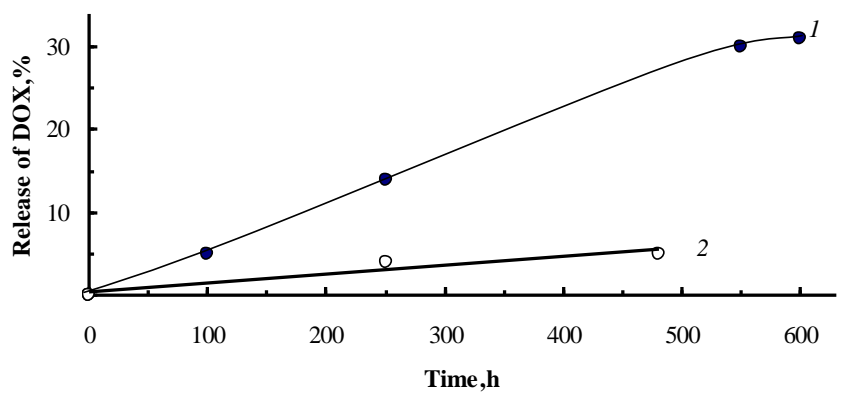

Fig. 7: Degree of release DOX from polymer conjugate at $\mathrm{pH}=4.6(1), 7.4$ (2).

\section{CONCLUSION}

Thus, in the course of the study the conjugate of DOX was synthesized, combining the drug and a vector for delivery of the drug. This compound is a promising, as it represents a new form of transport delivering a well-known and widely used anticancer agent DOX.

\section{Conflict of Interests}

The authors have no conflict of interests to disclose. In the publication used data obtained on the equipment of center for collective use "analytical center IMC RAS" (Nizhny Novgorod).
Acknowledgement: We are grateful for the assistance in obtaining the NMR spectra of the samples to Fedorov A. Yu., Professor of the Department of Organic Chemistry, Lobachevsky State University of Nizhny Novgorod and Malysheva Y.B., Associate Professor of the Department of Organic Chemistry, Lobachevsky State University of Nizhny Novgorod.

\section{REFERENCES}

Adams ML, Lavasanifar A, Kwon GS. Amphiphilic block copolymers for drug delivery. J Pharm Sci., 2003; 92(7): 1343-55.

Bae Y, Diezi TA, Zhao A, Kwon GS Mixed polymeric micelles for combination cancer chemotherapy through the concurrent delivery of multiple chemotherapeutic agents. J Control Release., 2007; 122: 324-30.

Bayazit MK, Clarke LS, Coleman KS, Clarke N. PyridineFunctionalized Single-Walled Carbon Nanotubes as Gelators for Poly(acrylic acid) Hydrogels. J. Am. Chem. Soc., 2010; 132: 15814-9.

Duncan R. Polymer conjugates as anticancer nanomedicines. Nat. Rev. Cancer., 2006; 6(9): 688-701.

Gabizon A, Shmeeda H, Barenholz Y. Pharmacokinetics of pegylated liposomal Doxorubicin: review of animal and human studies. Clin Pharmacokinet, 2003; 42: 419-436.

Gabizon AA. 2006. Liposomal Drug Carriers in Cancer Therapy. In Torchilin V.P., editors. Nanoparticulates as Drug Carriers. London: Imperiae College Pressp. P. 437-62.

Gann M.J., Higginbotham C.L., Geever L.M., Nugent M.J.D. The synthesis of novel pH-sensitive poly(vinyl alcohol) composite hydrogels using a freeze / thaw process for biomedical applications. Inter. J. Pharmaceut, 2009; 372 (1-2): 154-61.

Gaucher G, Marchessault RH, Leroux JC. Polyester-based micelles and nanoparticles for the parenteral delivery of taxanes. J Control Release, 2010; 143: 2-12.

Li C, Wallace S. Polymer-drug conjugates: Recent development in clinical oncologyAdv. Drug. Deliv. Rev., 2008; 60(8): 886-98

Nori A, Jensen KD, Tijerina M, Kopeckova P, Kopecek J.Subcellular trafficking of HPMA copolymer-TAT conjugates in human ovarian carcinoma cells. J Control Release, 2003, 91:53-9.

Salazar MD, Ratnam M. The folate receptor: what does it promise in tissue-targeted therapeutics? Cancer Metastasis Rev. 2007. 26 (1): 141-52. DOI:10.1007/s10555-007-9048-0

Yoo HS, Lee KH, Oh JE, Park TG. In vitro and in vivo antitumor activities of nanoparticles based on doxorubicin-PLGA conjugates. J Control Release, 2000; 68(3):419-31.

Xu Z, Guo M, Yan H, Liu K. Enhanced loading of doxorubicin into polymeric micelles by a combination of ionic bonding and hydrophobic effect, and the $\mathrm{pH}$-sensitive and ligand-mediated delivery of loaded drug. Reactive and Functional Polymers, 2013; 73: 564-72.

Wang Q, Xie X, Zhang X, Zhang J, Wang A. Preparation and swelling properties of $\mathrm{pH}$-sensitive composite hydrogel beads based on chitosan-g-poly(acrylic acid)/vermiculite and sodium alginate for diclofenac controlled release. Int. J. Biol. Macromol., 2010; 46:356-62.

Weissberger A, Proskauer ES, Riddick JA, Toops EE. 1955. Organic solvents. Physical properties and methods of purification: INC., New York, LTD., London.

Zhukova OV, Bulgakova SA. Synthesis of Polymer Derived Doxorubicin and Its Characterization by IR and UV Spectroscopy. Pharm Chem J, 2015; 48 (12): 813-7.

\section{How to cite this article:}

Zhukova O, Bulgakova S. Preparation of a Doxorubicin Conjugate with (Co) Polymers of Methacrylic acid for Targeted Tumor Therapy. J App Pharm Sci, 2016; 6 (07): 020-026. 